\title{
The emission of compact jets powered by internal shocks
}

\author{
Julien Malzac $^{1,2}$ \\ ${ }^{1}$ Université de Toulouse; UPS-OMP; IRAP; Toulouse, France \\ ${ }^{2}$ CNRS; IRAP; 9 Av. colonel Roche, BP 44346, F-31028 Toulouse cedex 4, France \\ email: Julien.Malzac@irap.omp.eu
}

\begin{abstract}
The emission of steady compact jets observed in the hard spectral state of X-ray binaries is likely to be powered by internal shocks caused by fluctuations of the outflow velocity. The dynamics of the internal shocks and the resulting spectral energy distribution (SED) of the jet is very sensitive to the shape of the Power Spectral Density (PSD) of the fluctuations of the jet Lorentz factor. I use Monte-Carlo simulations to investigate this dependence. It turns out that Lorentz factor fluctuations injected at the base of the jet with a flicker noise power spectrum (i.e. $P(f) \propto 1 / f$ ) naturally produce the canonical flat SED observed from radio to IR band in X-ray binary systems in the hard state. This model also predicts a strong, wavelength dependent, variability that resembles the observed one. In particular, strong sub-second variability is predicted in the infrared and optical bands.
\end{abstract}

Keywords. acceleration of particles, black hole physics, radiation mechanisms: nonthermal, methods: analytical, methods: numerical, radio continuum: stars, infrared: stars, X-rays: binaries

Steady compact jets are probably the most common form of jets in X-ray binaries. They appear to be present in all black hole and neutron star binaries when in the hard X-ray spectral state. They have an approximatively flat Spectral Energy Distribution (SED) extending from the radio to the mid-IR (e.g. Fender et al. 2000; Corbel \& Fender 2002; Chaty et al. 2003; Migliari et al. 2010). These flat spectra are usually ascribed to self-absorbed synchrotron emission from conical compact jets (Blandford \& Königl 1979) under the assumption of continuous energy replenishment of the adiabatic losses. The compensation of these energy losses is crucial for maintaining this specific spectral shape (Kaiser 2006).

Internal shocks provide a possible mechanism to compensate the adiabatic losses by dissipating energy and accelerating particles at large distance from the black hole. Internal shocks caused by fluctuations of the outflow velocity are indeed widely believed to power the multi-wavelength emission of jetted sources such as $\gamma$-ray bursts (Rees \& Meszaros 1994; Daigne \& Moscovitch 1998), active galactic nuclei (Rees 1978; Spada et al. 2001), or microquasars (Kaiser, Sunyaev \& Spruit 2000; Jamil et al. 2010). Internal shocks models usually assume that the jet can be discretised into homogeneous ejectas. Those ejectas are injected at the base of the jet with variable velocities and then propagate along the jet. At some point, the fastest fluctuations start catching up and merging with slower ones. This leads to shocks in which a fraction of the bulk kinetic velocity of the shells is converted into internal energy. Part of the dissipated energy goes into particles acceleration, leading to synchrotron and also, possibly, inverse Compton emission. Recently, however, Jamil et al. (2010) developed an internal shock model for the emission of jets in X-ray binaries, and concluded that energy dissipation through internal shocks only is not enough to produce a flat SED. Nevertheless, most studies of the internal shock model so far, including that of Jamil et al. (2010), have implicitly assumed that 

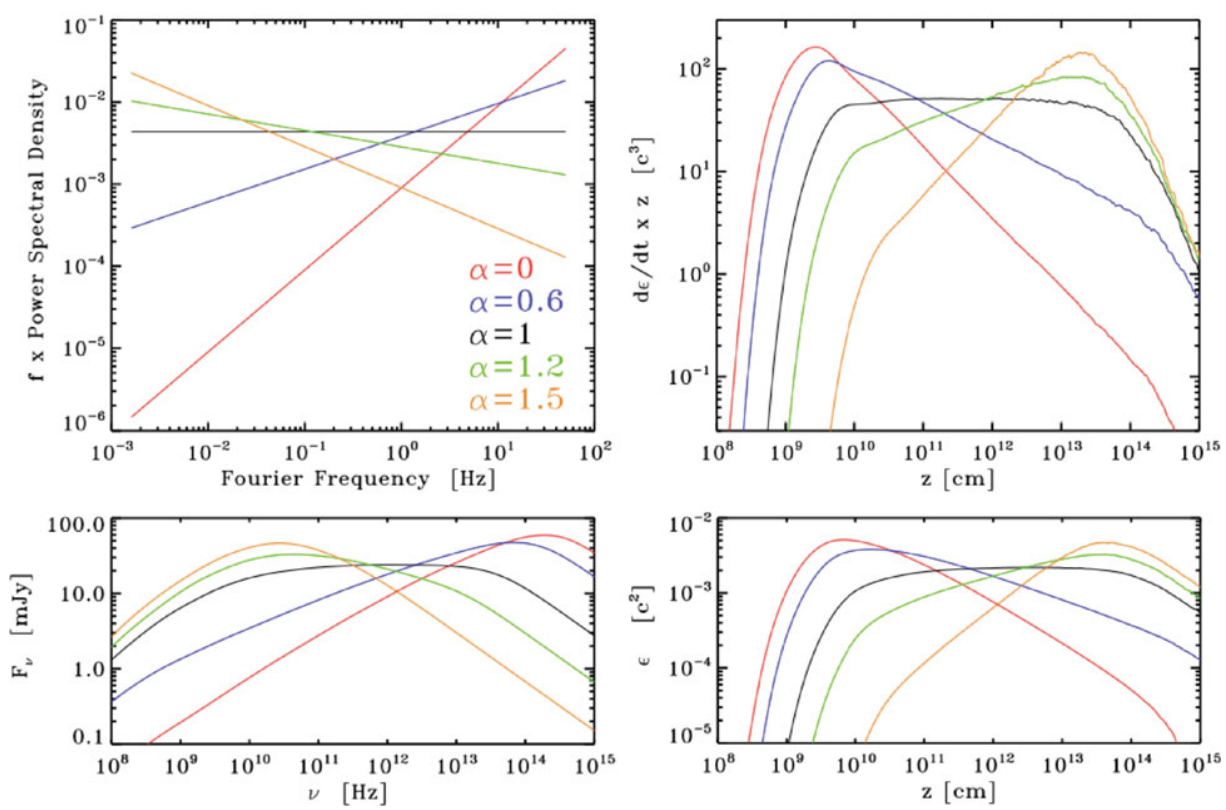

Figure 1. Simulation of the internal shock model with a power-law PSD of the Lorentz factor fluctuations $\left(P(f) \propto f^{-\alpha}\right)$. The top left panel shows the the shape of the injected PSDs, for the indicated values of the $\alpha$ index. Then moving clockwise other panels show the time averaged dissipation profile along the jet, the specific energy profile and the jet SED respectively. The SEDs are calculated for an inclination angle of 40 degrees and a distance to the source of $2 \mathrm{Kpc}$

the Fourier Power Spectral Density (PSD) of the velocity fluctuations injected at the base of the jet is flat (i.e. white noise). In fact, the energy dissipation profile of the internal shocks is very sensitive to the shape of the PSD of the velocity fluctuations. Indeed, let us consider a fluctuation of the jet velocity of amplitude $\Delta v$ occuring on a time scale $\Delta t$. This leads to the formation of a shock at a downstream distance $z_{s} \propto \Delta t / \Delta v$. In this shock the fraction of the kinetic energy converted into internal energy will be larger for larger $\Delta v$. From these simple considerations we see that the distribution of the velocity fluctuation amplitudes over their time scales (i.e. the PSD) is going to determine where and in which amount the energy of the internal shocks is deposited.

Here, instead of using white noise we use a more general model and assume that the PSD of the Lorentz factor of the jet has a power-law shape with index $\alpha: P(f) \propto f^{-\alpha}$. I used a code similar to that of Jamil et al. (2010) to explore the dependence of the photon spectrum on $\alpha$. Fig 1 shows that the dissipation profile along the jet and the profile of the specific energy of the flow are very sensitive to $\alpha$. For larger $\alpha$ the fluctuations of the Lorentz factor have, on average, longer time-scales and therefore more dissipation occurs at larger distances from the black hole. For the case $\alpha=0$ (i.e. white noise) we can see that most of the dissipation occurs very close to the black hole and then the dissipation rate decrease very quickly (like $z^{-5 / 3}$ ). As a consequence the specific energy profile is steep $\left(\propto z^{-2 / 3}\right)$ and therefore the adiabatic losses are not compensated. The photon spectrum is strongly inverted, with a slope $\simeq 0.65$ i.e more inverted than most of the observed spectra. On the other hand, one can see from Fig. 1 that for $\alpha=1$ (i.e. flicker noise) the dissipation profile scales like $z^{-1}$ and the specific energy profile is flat. In other words, the internal shocks compensate exactly for the adiabatic losses. As result the SED is flat over a wide range of photon frequencies. In fact, this result can 

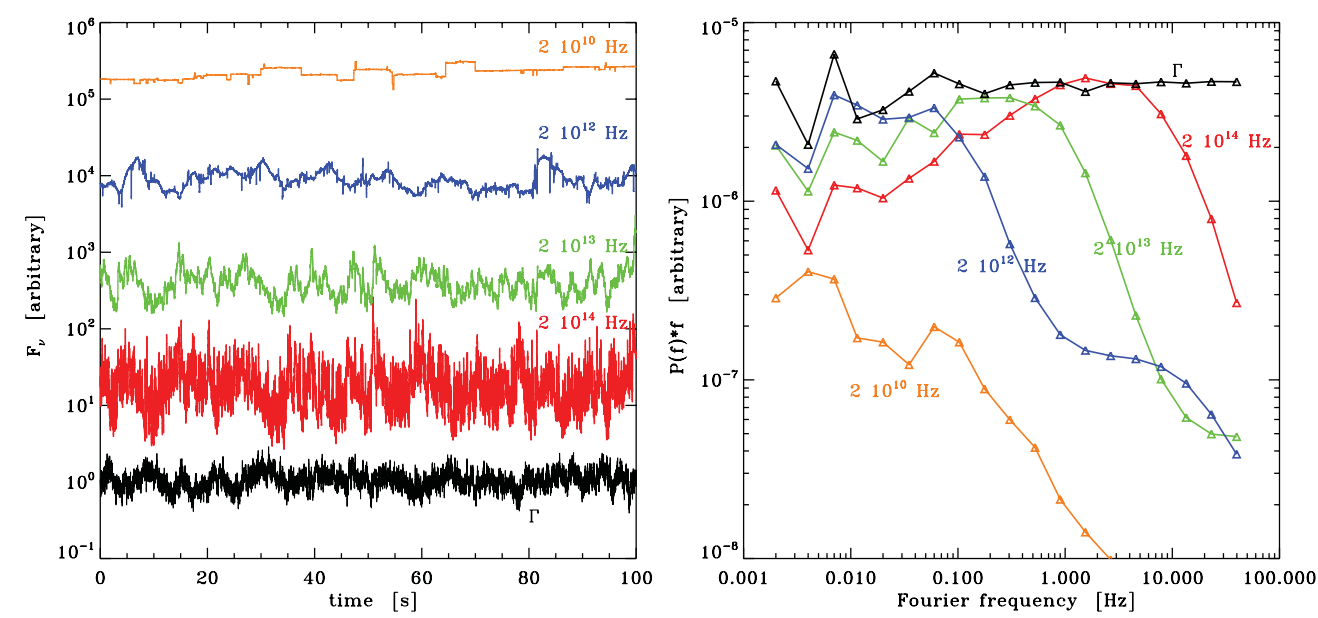

Figure 2. Synthetic light curves (left, rescaled) and power spectra at various indicated frequencies resulting from the simulation with $\alpha=0$. The injected fluctuations of the Lorentz factor are also shown.

also be obtained analytically (Malzac 2012). The case of flicker noise fluctuations of the jet Lorentz factor may therefore be relevant to the observations of compact jets.

In fact this type of fluctuations is not unexpected. Indeed, if the jet is launched by the accretion disc, the variability of the jet Lorentz factor must be related to that of the accretion disc. And we know, both from theory (see e.g. Lyubarskii 1995) and from observations (see e.g. Gilfanov \& Arefiev 2005) that accretion discs tend to generate flicker noise variability. Also, the highest and lowest frequencies of variability $f_{0}$ and $f_{1}$ must relate to time scales of the accretion flow. In the simulations show in Fig. 1, we choose $f_{1}=1.6 \times 10^{-3} \mathrm{~Hz}$ and $f_{0}=50 \mathrm{~Hz}$ corresponding to the Keplerian timescale at $10^{4} R_{g}$ and $10 R_{g}$ from a $10 M_{\odot}$ black hole respectively. We also assumed an average Lorentz factor of 2 varying with fractional rms amplitude of 30 percent, i.e. comparable to that observed in the X-ray variability of black hole binaries in the hard state. With these parameters, the dissipations starts at a distance of a few $10^{9} \mathrm{~cm}$. This is comparable to the distance of $\sim 10^{3} R_{g}$ inferred in Cyg X-1 both by the modelling the of SED (Zdziarski et al. 2012), and that of the orbital modulation of the radio emission (Zdziarski 2012). Our choice for the longest timescale of the injected fluctuations implies that significant dissipation occurs up to a distance of a about $10^{14} \mathrm{~cm}$ which is roughly comparable to the extension of the radio jet of Cyg X-1 in the VLBA images of Stirling et al. (2001). These authors also constrain the jet opening angle of Cyg X-1 to be small $\phi<2^{\circ}$. This implies that the radius of the jet at the base of the emitting region is at most of a few $10^{8} \mathrm{~cm}$. We assumed that the jet power is of the oder of a few percent of the Eddington luminosity $L_{E}$ as infered in Cyg X- 1 (Gallo et al. 2006). This implies an equipartition magnetic field at the base of the emitting region, of the order of $10^{4}$ gauss. These estimates for the jet radius and magnetic field at the base of the dissipation region are comparable to the values inferred in Cyg X-1 (Zdziarski et al. 2012), GX 339-4 (Gandhi et al. 2011) and XTE J1550-564 (Chaty et al. 2011). The SEDs of Fig. 1 are evaluated assuming that the synchrotron emitting electrons have a power law energy distribution $n\left(\gamma_{e}\right) \propto \gamma_{e}^{-2.3}$ for electrons Lorentz factors, $\gamma_{e}$, in the range $1-10^{6}$. This estimate is consistent with the flux observed in bright hard states (e.g. at $\simeq 2 \mathrm{kpc}, \mathrm{Cyg} \mathrm{X}-1$ has an average radio flux of of 15 $\mathrm{mJ}$ at $15 \mathrm{GHz}$ ). The turnover frequency appears in the mid-infrared $\nu_{t} \simeq 2 \times 10^{13} \mathrm{~Hz}$, in agreement with the observations of Cyg X-1 (Rahoui et al. 2011) or GX 339-4 (Gandhi 
et al. 2011): Finally the model predicts that the emission should decrease significantly at frequencies below $\nu_{s} \sim 1 \mathrm{GHz}$. The specific value of $\nu_{s}$ depends on $f_{1}$ and this is something that can be investigated in the near future with LOFAR.

An interesting feature of the internal shock model is that it naturally predicts strong variability of the the jet emission. Figure 2 shows sample light curves and power spectra obtained from the simulation with $\alpha=1$. The jet behaves like a low-pass filter. As the shells of plasma travel down the jet, colliding and merging with each other, the highest frequency velocity fluctuations are gradually damped and the size of the emitting region increases. The jet is strongly variable in the optical and IR bands originating primarily from the base of the emitting region and become less and less variable at longer frequencies produced at larger distances from the black hole. The observations also show significant flickering in the Infrared and optical band (Kanbach et al. 2001; Casella et al. 2010; Gandhi et al. 2010). At least part of of this fast IR/optical variability is likely to arise from the jet, possibly through internal shocks. about $100 \mathrm{~ms}$ between the X-ray and IR light curves of GX 339-4, a similar time lag appears to be present, also between optical and a time lag of about $100 \mathrm{~ms}$ is detected in

Internal shocks driven by flicker noise fluctuations can therefore produce not only the flat SED of compact jets in X-ray binaries, but also other properties such as the flux amplitude or the location of the break frequency. The model also predicts strong multiwavelength variability that appear to be similar to that observed.

\section{References}

Blandford, R. D. \& Königl, A. 1979, ApJ, 232, 34

Casella, P., et al. 2010, MNRAS, 404, L21

Chaty, S., et al. 2003, MNRAS, 346, 689

Chaty, S., Dubus, G., \& Raichoor, A. 2011, A\&A, 529, A3

Corbel, S. \& Fender, R. P. 2002, ApJ, 573, L35

Daigne, F. \& Mochkovitch, R. 1998, MNRAS, 296, 275

Fender, R. P., et al. 2000, MNRAS, 312, 853

Gallo, E., et al. 2005, Nature, 436, 819

Gandhi, P., et al. 2010, MNRAS, 407, 2166

Gandhi, P., et al. 2011, ApJ, 740, L13

Gilfanov, M. \& Arefiev, V. 2005, astro, arXiv:astro-ph/0501215

Jamil, O., Fender, R. P., \& Kaiser, C. R. 2010, MNRAS, 401, 394

Kaiser, C. R., Sunyaev, R., \& Spruit, H. C. 2000, A\& $A, 356,975$

Kaiser, C. R. 2006, MNRAS, 367, 1083

Kanbach, G., Straubmeier, C., Spruit, H. C., \& Belloni, T. 2001, Nature, 414, 180

Lyubarskii, Y. E. 1997, MNRAS, 292, 679

Malzac 2012, MNRAS, in press.

Migliari, S., Miller-Jones, J. C. A., \& Russell, D. M. 2011, MNRAS, 415, 2407

Rahoui, F., et al. 2011, ApJ, 736, 63

Rees, M. J., 1978, MNRAS, 184, 61P

Rees, M. J., Meszaros, P. 1994, ApJ, 430, L93

Spada, M., Ghisellini, G., Lazzati, D., \& Celotti, A. 2001, MNRAS, 325, 1559

Stirling, A. M., et al. 2001, MNRAS, 327, 1273

Zdziarski, A. A. 2012, MNRAS, 422, 1750

Zdziarski, A. A., Lubiński, P., \& Sikora, M. 2012, MNRAS, 2816 